\title{
MODELS OF BIPOLAR MOLECULAR OUTFLOWS
}

\author{
SYLVIE CABRIT \\ DEMIRM, Observatoire de Paris \\ 61 Avenue de l'Observatoire \\ 75014 Paris, France \\ ALEX RAGA \\ Instituto de Astronomía, UNAM, Ap. 70-264 \\ 04510 México D. F., México \\ AND
FREDERIC GUETH
IRAM, 300 rue de la Piscine
38406 Saint Martin d'Hères, France
}

\begin{abstract}
This paper reviews various hydrodynamical models proposed for explaining bipolar molecular outflows from young stars. The different possibilities discussed are : wind-driven molecular shells, steady state filled flows, and jet-driven bow shocks. Particular emphasis is placed on comparisons between model predictions and observations of molecular flows. While it appears that jet-driven bow shocks are at the present time the most popular mechanism for explaining molecular outflows, other models have interesting characteristics that might be relevant for at least some objects.
\end{abstract}

\section{Introduction}

Bipolar molecular outflows from young stellar objects (YSOs) are a crucial diagnostic of mass-loss properties during the earliest stages of stellar evolution. They contain unique information about a number of fundamental parameters of the primary wind that created them, including not only its global energetics (Lada 1985; André, this volume), but also its velocity, intrinsic collimation, and time variability (in both velocity and direction), which could set strong constraints on the wind ejection mechanism. In ad- 
dition, molecular outflows appear to have a large impact on the chemistry and turbulence of the interstellar medium. They might also play a role in dispersing infalling envelopes around YSOs. Addressing these questions requires modelling the acceleration process and long term dynamical evolution of the flows.

A number of observational properties seem to characterize molecular outflows and are useful to constrain models. Recent summaries are given e.g. in Masson \& Chernin (1993), Bachiller (1996), and Lada \& Fich (1996). They include: (1) average line shape indicating a power-law mass distribution with velocity $m(v) \propto v^{-\gamma}$ with $\gamma \sim 1.3-2.1$ (Rodríguez et al. 1982; Masson \& Chernin 1992; Stahler 1994), (2) moderate collimation with initial opening angles $\sim 20^{\circ}-90^{\circ}$ and apparent length-to-width ratios of 3-10; (3) limb-brightened cavities at low-velocity, and higher collimation at high velocity; (4) apparent linear acceleration ('Hubble-law') in flows with wellseparated outflow lobes; (5) rare occurence of overlapping blueshifted and redshifted emission within the same flow lobe, implying only a small amount of transverse motion (Cabrit et al. 1988; Meyers-Rice \& Lada 1991); (6) in some cases, extremely high-velocity (EHV) features reaching $150 \mathrm{~km} / \mathrm{s}$, seen along the flow axis in $\mathrm{CO}$, vibrationally excited $\mathrm{H}_{2}$, and shock-enhanced species like $\mathrm{SiO}$ or $\mathrm{CH}_{3} \mathrm{OH}$ (cf. Bachiller \& Pérez Gutiérrez, this volume).

Proposed dynamical models can be separated into three broad classes: (1) molecular shells driven by wide-angle winds, (2) steady-state filled flows with internal stratification, (3) jet-driven molecular bow shocks. The present review will summarize their strong and weak points, and mention further developments that would help to distinguish between them.

\section{Molecular shells driven by wide-angle winds}

The first hypothesis for the generation of bipolar molecular outflows involved an initially isotropic wind blowing into a stratified molecular environment, resulting in the formation of bipolar swept-up shells (Snell et al. 1980). This was supported by the typically low momentum flux in ionized components of YSO jets, requiring an additional neutral component to drive molecular flows (Mundt et al. 1987; Lizano et al. 1988). While there is now clear evidence for collimated, high-velocity neutral gas that could solve the "jet momentum problem", wind-driven shells remain attractive to explain flows of large lateral extents and low collimation.

The expansion of wind-driven shells has been the subject of detailed analytical studies (e.g. Koo \& McKee 1992 and refs. therein). In the case of molecular flows from YSOs, any mixing with ambient molecular gas should strongly enhance wind cooling, and the shells are often assumed to be in the highly radiative "momentum-driven" regime (even though the nominal 
criterion may not always hold; Frank \& Mellema 1996). Hot wind bubbles seem excluded as they would predict too much sideways expansion (Masson \& Chernin 1993). The next sections describe two analytical models of momentum-conserving wind-driven outflows, and recent numerical results.

\subsection{RADIALLY EXPANDING MOMENTUM-DRIVEN SHELLS}

Moriarty-Schieven \& Snell (1988) and Meyers-Rice \& Lada (1991) showed that properties of the L1551 and Mon R2 flows can be empirically reproduced by a thin shell with higher velocity toward the more distant parts. Shu et al. (1991) proposed a simple, self-similar dynamical model relying on radial expansion of the swept-up shell (i.e., assuming that shocked wind and ambient material instantaneously mix inside the shell, resulting in a net outward momentum in the same radial direction as the wind). Ambient density varies with radius as $1 / r^{2}$, cancelling the decrease in wind ram pressure due to radial expansion, so that the shell velocity is constant in time (for fixed polar angle $\theta$ ), but variations of density and wind thrust as a function of $\theta$ produce faster expansion along the axis and formation of bipolar lobes. At any given time $t, V(\theta)=t \times r(\theta)$ so that more distant parts of the shell also have a larger velocity, creating the observed 'Hubble law'. The cavity maintains the same shape and degree of collimation as its expands.

Masson \& Chernin (1992) pointed out a potential problem of too much mass at high velocity. They found that the steep observed mass spectrum $m(v) \propto v^{-1.8}$ in molecular flows required loosely collimated winds and nearly evacuated polar regions, contrary to usual expectations. Modelling of the CO intensity in a cavity structure in the Mon R2 outflow yields the same result (Xie et al. 1993). However, Li \& Shu (1996) noted that such thrust and density distributions are naturally encountered in models of MHD winds from the stellar/disk magnetosphere (Shu et al. 1995) and of self-similar critical magnetized cores. The global cavity shape and average $m(v)$ are then consistent with observations. Furthermore, the uncomfortable need for two distinct mass-loss components (a wind and a jet) can be avoided by interpreting the jet as a mere axial density enhancement in the MHD wind (see also Shu \& Shang, this volume).

This simple model still meets some limitations. To reproduce the largest flows, e.g. Mon R2, the appropriate density law would have to hold over distances reaching a few pc. Another problem with radially expanding thin shells is the predicted velocity range at low inclination (cf. Cabrit \& Bertout $1986 ; 1990)$. As an illustration, in Figure 1 we show position-velocity (P-V) diagrams calculated for a wind thrust per solid angle $F_{w} \propto 1 / \sin ^{2} \theta$ and ambient density $\rho_{e} \propto \sin ^{2} \theta\left(1+1.14 \sin ^{2} \theta\right) / r^{2}$ (close to the $n=2$ solution 


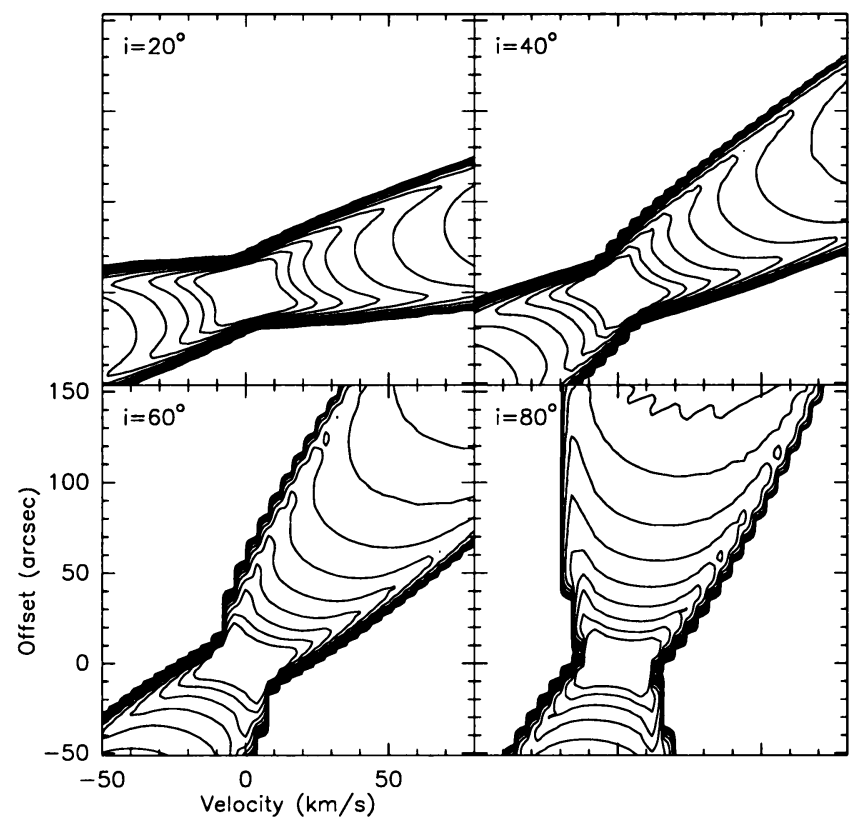

Figure 1. Synthetic position-velocity diagrams at various angles $i$ to the line of sight for a radial wind-driven shell model similar to that of $\mathrm{Li} \& \mathrm{Shu}$ (1996), assuming isothermal optically thin $\mathrm{CO}$ emission. Contours increase by factors of 2 .

from $\mathrm{Li} \& \mathrm{Shu}$ 1996). The two ridges trace the front and back side of the shell. At large angles to the line of sight $i>70^{\circ}$, the back side is projected close to zero velocity, and the diagram agrees with that seen in many flows such as NGC2264-G (Lada \& Fich 1996) or HH111 (modelled as a radially wind-driven shell by Nagar et al. 1997). Unfortunately, it also resembles the diagram predicted for bow shocks (see Figure 3) so that there is no unambiguous interpretation.

At lower inclination $i<70^{\circ}$, most of the shell becomes blueshifted and has no more low-velocity emission (while it is still present in a bow shock, cf. Fig. 3). Such line profiles 'detached' from cloud emission are not frequently reported. Examples are Mon R2 (Meyers-Rice \& Lada 1991) and TMC1 and TMC1A (Chandler et al. 1996). However, the latter may suffer from subtraction of low-velocity CO emission from the off-position. Shockenhanced molecules, being free from confusion by ambient gas but affected by velocity-dependent chemistry, provide complementary information on the velocity range in shocked regions of outflows. In L1448, redshifted $\mathrm{SiO}$ emission extends all the way down to zero velocity, apparently more compatible with a bow shock (Guilloteau et al. 1992; Dutrey et al. 1997). Al- 
ternatively, wind-driven shells might have more low-velocity emission if the shell is thick and turbulent. More data on flows at low inclination would be useful to further assess this problem.

Finally, the shell in the Li \& Shu model does not close back on axis. The polar regions would be dominated by a broad 'cap' of wind material, possibly shocking against itself to produce the multiple EHV features. It is not yet known whether it could reproduce the geometry and kinematics seen e.g. in $\mathrm{H}_{2}$ images of young flows (e.g. McCaughrean et al. 1994).

\subsection{STEADY WIND-BLOWN CAVITIES}

Steady bipolar configurations may exist where the shocked wind is in pressure balance with the ambient medium. In the adiabatic case, transonic de Laval nozzles are established (Königl 1982; Smith et al. 1983). In the strongly cooling case, perhaps more relevant for YSOs, the wind refracts through an oblique shock and slides supersonically along the cavity walls, exerting a centrifugal pressure that contributes to balance the ambient pressure (e.g. Cantó 1980). Bipolar cavities with acute tips can be formed for a broad range of ambient pressure laws $P \propto r^{-n}$, with $0 \leq n<2$ (Barral \& Cantó 1981; Smith 1986). In particular, the Barral \& Cantó model shows surprisingly good agreement with ovoid cavities observed at high resolution in $\mathrm{CO}$ and $\mathrm{H}_{2}$ in the L1157 and HH46/47 outflows (Gueth et al. 1996; Eislöffel et al. 1994), including the presence of terminal shocks.

Steady state cavities would alleviate wind momentum requirements, as the flow could be older than its dynamical time (Dyson et al. 1988). However, they encounter several problems: (1) as material flows out parallel to the surface and refocusses toward the axis, projection of the front and back sides of the shell creates an X-shaped pattern in P-V diagrams which is not consistent with observations; (2) The presence of cavities at different orientations in the same flow, like in L1157, is not easily explained (it is in fact a problem for all wind-driven shells); (3) finally, shocked wind material that converged at the tip of the cavity should form a dense highvelocity jet (Cantó et al. 1988) which would presumably create a separate, non-stationary, jet-driven outflow.

\subsection{NUMERICAL SIMULATIONS}

The above wind-driven shell models easily reproduce outflow shapes, but the predicted kinematics are not fully satisfying. This may be due in part to the simplifying extreme assumptions: full mixing in the radially expanding model, full steady-state, instant cooling, infinitely thin shell.

Detailed numerical simulations have only been performed quite recently, and they show that the actual situation could be more complex than anti- 
cipated. Adiabatic simulations with an ambient stratification similar to that of Li \& Shu (1996), although with density decreasing as $\rho \propto r^{-1.5}$, reveal a mixture of bubble and jet behavior, as the hot wind is focussed through a non-spherical inner shock (Frank \& Mellema 1996; see also this volume). The strongly cooling case produces strong convergence toward the axis, as in the steady-state solution of Barral \& Cantó (1981), but also complex vortical motions at the wind/ambient interface (Mellema \& Frank 1995). Such studies must be pursued to allow realistic comparisons between the dynamics of wind-driven cavities and molecular outflow observations.

\section{Steady-state filled flows}

Low-velocity 'cavities' could readily be obtained at all view angles if the outflow is not restricted to a thin shell but fills the lobe volume and has a velocity decreasing toward the flow edges (Levreault 1988; Cabrit \& Bertout 1990). Several dynamical models predict such an internal structure.

\subsection{TURBULENT MIXING-LAYERS}

Kelvin-Helmholtz instabilities along the jet/environment boundary lead to the formation of a turbulent viscous mixing layer that grows both into the environment and into the jet. Eventually the whole flow becomes turbulent (recent numerical simulations for cooling jets are presented by e.g. Massaglia et al. and Stone, this volume, and Downes, 1997). The possibility that molecular outflows are turbulent layers entrained around YSO jets has been investigated in several analytical studies.

Stahler (1994) considered a fully turbulent flow of arbitrary structure. He pointed out that the mass distribution with velocity $m \propto v^{-\gamma}$ could result from a velocity cross-section of the form $v \propto r^{-2 /(\gamma-1)}$, while the observed acceleration would be an artifact of growing flow thickness at larger distances; however there was no self-consistent dynamical justification for the transverse profiles and flow sizes.

Cantó \& Raga (1991) derived a "mixing-length" parameterization of the turbulent viscosity, calibrated with laboratory experiments for jet Mach numbers $M_{j}=1-20$, that allowed them to derive the growth of the mixinglayer width with distance. However, for the isothermal case relevant for radiative YSO jets, the layer is too narrow, producing outflows that have length-to-width ratios $q$ of $\sim 20$, approximately 5-10 times larger than the ones observed in molecular outflows. Different parameterizations of the turbulence were proposed by Lizano \& Giovanardi (1995) and Dyson et al. (1995), but as no calibrations were carried out for these parameterizations, it is not possible to use them for determining the flow opening angles. 
Raga et al. (1993) showed that turbulent mixing-layers could be broadened by sideways ejection of overpressured jet material at velocity discontinuities along the jet. The overall properties agree much better with molecular flows. However, $q$ would still be very high, $\geq 10$, compatible only with the most collimated outflows known to date. This problem might be alleviated by the presence of a strong pressure gradient in the surrounding environment (Richer et al. 1992). Another drawback is the predicted velocity gradient: after providing strong acceleration where the first working surface forms, the ejected jet momentum is progressively shared among larger and larger amounts of ambient gas, and the mean velocity in the turbulent envelope rapidly decreases away from the star. In contrast, most molecular outflows show velocities linearly increasing with distance from the star, followed by sudden deceleration (e.g. L1448, Bachiller et al. 1990).

Therefore, turbulent entrainment as currently modelled does not explain all properties of molecular outflows. It is not clear whether models with less drastic simplifying assumptions might show better agreement with observations. Nevertheless, chemical and thermal balance calculations show that molecules could survive in jet mixing layers, and produce collimated emission at high velocities (Taylor \& Raga 1995). $\mathrm{H}_{2}$ images suggest this process is active, at least close to the jet injection point (e.g. Gredel \& Reipurth 1993; Noriega-Crespo, this volume). It would also be an attractive explanation for the EHV CO jets observed in some flows such as Orion B and HH211 (Richer et al. 1992; Guilloteau et al., this volume), the alternative explanation being that the jet is intrinsically molecular.

\subsection{GLOBAL INFALL-OUTFLOW PATTERN}

A new approach was recently proposed by Henriksen \& Valls-Gabaud (1994) and fully developed by Fiege \& Henriksen (1996a): the bipolar outflow is not entrained by an underlying wind or jet, but it traces infalling matter that has been deflected toward the poles in a central torus of high MHD pressure, and accelerated above escape speeds by local heating. The magnetic/stream lines are quadrupolar. The self-similar outflow solution has decreasing speed and increasing mass at larger angles from the axis up to $\theta_{\text {max }} \sim 50^{\circ}$, nicely reproducing the observed conical cavities, increasing collimation at higher velocity, decreasing line wings, and apparent acceleration with distance (Fiege \& Henriksen 1996b). However, the model has several limitations: radiative acceleration requires unrealistically high central luminosities $\sim 10^{5} L_{\odot}$, suggesting that Poynting flux driving or local dissipative heating should be included. The fastest axial regions are not part of the self-similar solution, and will require special treatment.

Independently of further refinements, general model features may be ac- 
cessible to observational tests. Sub-mm and mid-IR polarimetry could set constraints on a global quadrupolar magnetic configuration (e.g. Holland et al. 1996). More important, mass-flux through the outflow should be entirely provided by infall and directly related to the mass in the thick torus. Relevant data are becoming available for such a comparison (e.g. Fuller et al. 1995; Bontemps et al. 1996).

\section{Jet-driven bow shocks}

Recent observational findings have made this model increasingly attractive, in particular (1) the low ionized fraction in optical jets (e.g., Hartigan et al. 1994), and the highly collimated EHV atomic and molecular features in some flows (e.g. Bachiller et al. 1990; Masson et al. 1990; Richer et al. 1992), both indicate that jets may have enough momentum for driving outflows; (2) the close spatial association of curved shocks seen in $\mathrm{H}_{2}$ and shock-enhanced species $\left(\mathrm{SiO}, \mathrm{NH}_{3}\right)$ with local acceleration in the sweptup CO (e.g. Davis \& Eislöffel 1995), and with the tip of CO cavities in interferometric maps (e.g. L1157, Gueth et al. 1996), is strongly suggestive of jet working surfaces.

\subsection{GENERAL PROPERTIES OF BOW SHOCKS}

Bow Shocks have been extensively studied to model optical properties of Herbig-Haro objects (see Raga 1995, and references therein). The structure of a bow shock at the head of a jet is illustrated in Figure 2. Across the jet, a working surface is formed where jet and ambient gas are arrested in a twoshock layer. High pressure gas between the shocks is ejected sideways out of the jet beam; it then interacts with unperturbed ambient gas (flowing back at $-V_{B S}$ in the frame of the shock) through a broader shock surface (the bow shock); the swept-up ambient gas is shocked at an oblique angle and streams back parallel to the bow surface in a dense layer. Some postshock gas reexpands in the low-pressure region behind the bow shock, creating a low-density cocoon with mixed ambient and jet material. Bow Shocks are appealing, as they readily explain several observed properties of molecular flows (see e.g. the analytical studies of Masson \& Chernin 1993; Raga \& Cabrit 1993):

(1) they naturally create an apparent acceleration: As one moves from the broader wings to the narrow apex of the bow shock the shock becomes less oblique, and the net forward velocity increases (up to $V_{B S}$ at the bow shock apex). (2) There is more swept-up mass at low velocities: the intercepted mass flux is determined by the bow shock cross-section, which steadily grows in the bow wings while velocity decreases. 

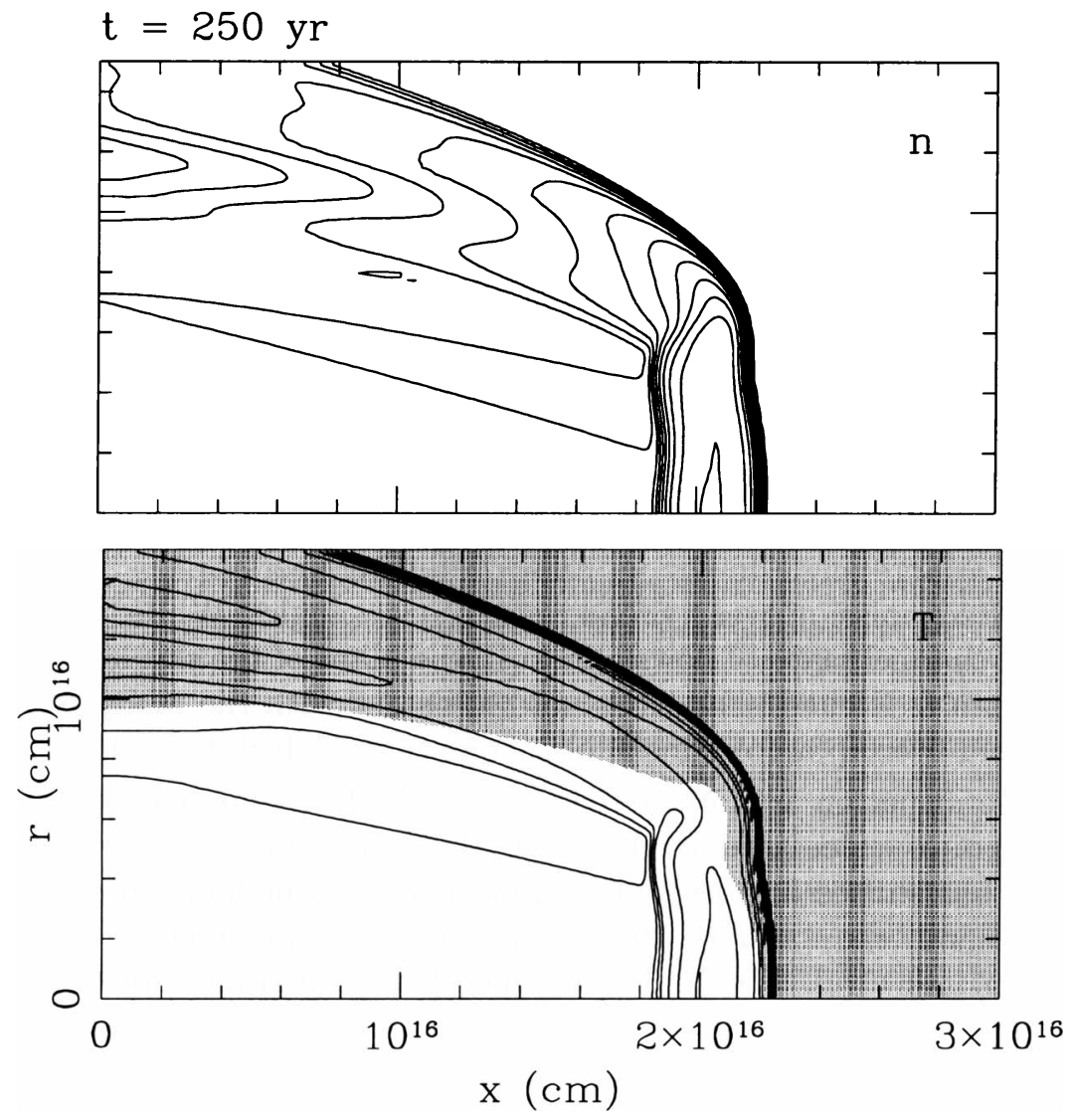

Figure 2. High-resolution 2D numerical simulation of the bow shock at the head of a jet. Top panel shows density and bottom panel temperature. Contours increase by factors of 2. The shaded area shows the locus of ambient gas. From Raga \& Cantó (1997)

Recently, efforts have been made to simulate jet bow shocks with conditions more appropriate for molecular outflows (SPH with atomic cooling: Chernin et al. 1994; 2D high resolution grid with $\mathrm{H}_{2}$ destruction and cooling: Raga et al. 1995; 2D slab jet with $\mathrm{H}_{2}$ : Downes 1996). An important result is that $\mathrm{H}_{2}$ and $\mathrm{CO}$ molecules can survive dissociation in the bow shock (cf. Smith et al. 1991; Wolfire \& Königl 1991), producing molecular emission at velocities up to $30-60 \mathrm{~km} / \mathrm{s}$ (e.g. Raga al. 1995). The most complete exploration so far is that of Suttner et al. (1997), who present simulations of the whole flow (from $1 \mathrm{D}$ to $3 \mathrm{D}$ ) including $\mathrm{H}_{2}$ chemistry and cooling, and consider high densities $\sim 10^{4}-10^{5} \mathrm{~cm}^{-3}$ as well as the effect 
of jet pulsing and precession. The $\mathrm{H}_{2}$ emission maps, as well as the $\mathrm{CO}$ line profile shape, P-V diagram, and channel maps show remarkable agreement with observations (Smith et al. 1997).

Despite their successful aspects, Lada \& Fich (1996) argued that bow shocks may meet two problems: they would be expected to produce too much sideways expansion, and lobes that are too narrow. We discuss solutions to these two important issues in the following sections, and in particular investigate the effects of jet time variability on the flow structure.

\subsection{SIDEWAYS MOTIONS IN BOW SHOCKS}

A bow shock pushes ambient material into a motion normal to the surface of the shock. In the oblique bow shock wings, the postshock velocity vector makes a large angle to the jet axis, producing overlapping blueshifted and redshifted emission over a broad range of view angles. While this description can fit channel maps of flows almost in the plane of the sky, such as RNO 43 (Padman \& Richer 1994), it is not consistent with the rarity of significant blue/red overlap among molecular flows (e.g. Cabrit \& Bertout 1986).

The situation is not so clear cut in hydrodynamical simulations: PV diagrams calculated by Raga et al. (1995) do show substantial overlap of blueshifted and redshifted emission at an inclination of $45^{\circ}$. On the other hand, the lower resolution 3D simulations of Chernin et al. (1994) and Suttner et al. (1997) show a much better alignment of the velocity of emitting material with the symmetry axis of the flow. Smith et al. (1997) show (through a simple, analytic model) that the higher forward velocities of emitting gas could arise from turbulent mixing inside the narrow, cold swept-up layer. Emission then includes contribution of material that went through the bow shock closer to the jet head, and has a larger forward momentum. The result is somewhat equivalent to the intuitive global momentum conservation argument used by Masson \& Chernin (1993). Higher forward velocities could also be obtained through mixing of postshock ambient gas with jet gas, a process investigated under simplifying assumptions by Raga, Cabrit \& Cantó (1995).

Interesting in this context is the stellar wind bow shock model of Wilkin (1996; see also Wilkin et al., this volume) which assumes full mixing between postshock ambient and wind gas, and solves exactly for the bow shock shape and velocity. This flow is qualitatively similar to the flow found around an internal working surface (see Raga \& Cabrit 1993). In the model of Wilkin (1996) the layer velocity depends on an extra parameter $\alpha=V_{B S} / V_{w}$ where $V_{B S}$ is the bow shock velocity and $V_{w}$ the wind velocity (i.e. in the present context the velocity of the material ejected from the working surface, which is approximated as an isotropic wind source). The final opening angle of ve- 


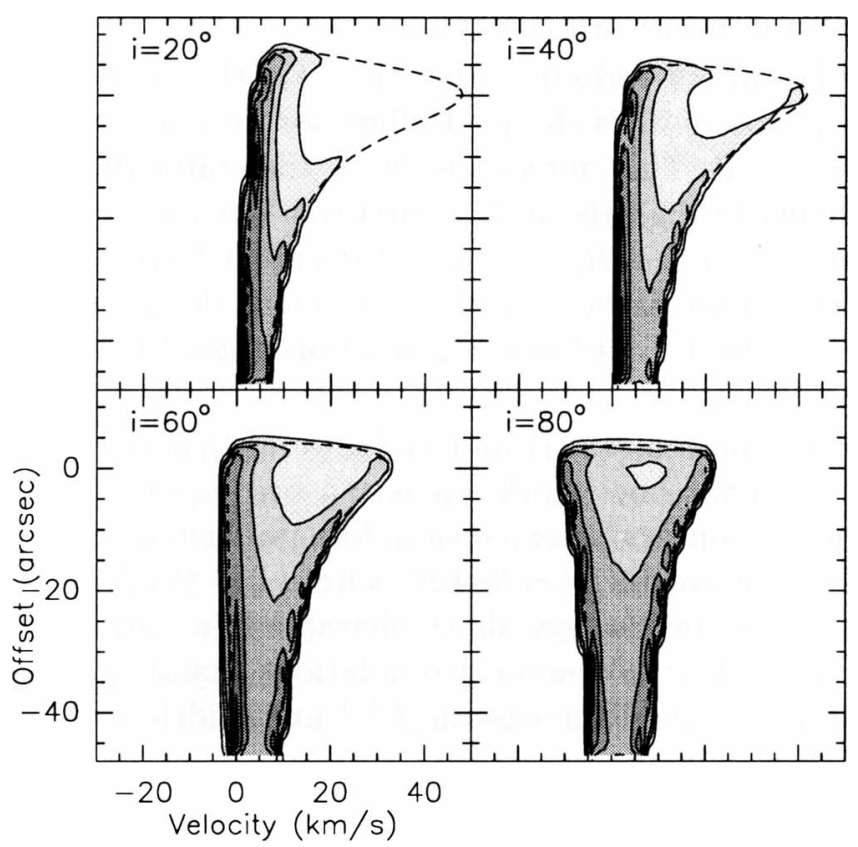

Figure 3. Synthetic position-velocity diagrams at various inclinations $i$ to the line of sight for the analytical bow shock model of Wilkin (1996), assuming similar bow shock and wind velocities $V_{B S}=V_{w}=50 \mathrm{~km} / \mathrm{s}$, and isothermal optically thin CO emission.

locity vectors in the bow shock wings $\theta_{\max }$ decreases for larger $\alpha$ (Wilkin et al. 1997). P-V diagrams for $\alpha=1\left(\theta_{\max } \sim 40^{\circ}\right)$ are shown in Figure 3. Negative velocities are essentially absent until $i \geq 50^{\circ}$. However, low-velocity emission remains present at all view angles, in contrast with P-V diagrams of wind-driven shells in Fig. 1.

One must mention another situation leading to more forward-directed velocities, namely when the bow shock propagates into material already moving away from the source, as expected for internal working surfaces produced in a jet from a variable source (cf. Sect. 4.4.1). In this case, the post-bow shock velocity vector is no longer perpendicular to the surface, and is oriented closer to the outflow axis (see e. g. Hartigan et al. 1987).

\subsection{WIDTH AND COLLIMATION OF JET-DRIVEN BOW SHOCKS}

Bow shocks may produce conical flow lobes toward the star, both because of the initial transient after jet injection (while the bow shock grows to its full size), and because of considerably reduced expansion in the much denser environment around the protostar (Masson \& Chernin 1993; Raga 
\& Cabrit 1993; cf. Fig. 4). On the other hand, the low collimation and large flow radii do not seem as straightforward to explain.

Hydrodynamical simulations of radiative bow shocks yield maximum lobe radii of $R_{c} \sim 3-5 r_{j}$ (where $r_{j}$ is the jet radius) for lobe lengths of $L \sim 25-80 r_{j}$, hence $q \sim 4-8$. This agrees well with the collimation of outflows observed at sufficiently high resolution. However the inferred jet radii would be very large, e.g. $r_{j} \sim R_{c} / 4 \sim 7 \times 10^{16} \mathrm{~cm}$ in NGC2264G (Lada \& Fich 1996). Furthermore, as bow shocks grow more slowly in the transverse direction than in the forward one, collimation should increase over time.

In particular, Masson \& Chernin (1993) and Wilkin (1996) noted that for strong cooling, material in the bow shock wings can be described as a shell undergoing a momentum-conserving expansion. In a uniform medium, a cubic asymptotic shape is obtained : $R_{c}^{3} \sim 3 \pi L R_{o}^{2}$ where $L=t V_{B S}$ is the distance from the apex, and $R_{o}$ is the bow shock characteristic radius for the head of a jet, the above hydrodynamical simulations would suggest $R_{o} \sim 0.4 r_{j}$. Hence collimation should increase as $L^{2 / 3}$ and width as $L^{1 / 3}$ only. Several possible solutions may be invoked:

1- Some molecular lobes may trace the 'truncated' inner regions of a much longer jet. HH111 is a possible example: while the CO cavity is only $80^{\prime \prime}$ long, the full jet stretches over $L \sim 367^{\prime \prime}$. For the measured jet radius $r_{j} \sim 1.5^{\prime \prime}$, the above cubic approximation would predict $R_{c} \sim 11^{\prime \prime}$, as observed (Cernicharo \& Reipurth 1996). The increasing number of parsecscale Herbig-Haro flows, and of faint molecular lobes beyond previously mapped areas (see contributions by Bally \& Devine and by Padman et al., this volume) further supports this possibility. However, broader flows with $R_{c} \sim 2.5 \times 10^{17} \mathrm{~cm}(\mathrm{NGC} 2264 \mathrm{G}, \mathrm{L} 1551)$ would require very long jets (>7 pc!) if $r_{j} \leq 2 \times 10^{16} \mathrm{~cm}$.

2- Time variability in jet velocity could produce new, shorter cavities inside the old one, and possibly increase the outflow width by sideways ejection of material from the jet (Raga \& Cabrit 1993).

3 - Jets may have an 'effective radius' that grows with distance roughly as $L / 20-L / 40$, maintaining a low bow shock collimation $q \sim 3-5$. This could happen through jet wandering in direction (Masson \& Chernin 1993), and/or through growth of a viscous mixing-layer (cf. Sect. 3.1).

Observations indeed bring growing evidence for time variability in flows, and we explore its effect in more detail in the next section.

\subsection{EFFECT OF JET TIME VARIABILITY}

Herbig-Haro jets often contain multiple bow shocks; similarly, molecular flows often present successive EHV peaks roughly equally spaced along the 
flow axis, with point symmetry between blue and red lobes suggesting that they originate from intrinsic variability at the source (e.g. L1448, Bachiller et al. 1990; RNO 43, Bence et al. 1996). Recent interferometric imaging confirms the presence of multiple cavities, sometimes with differing orientations (Gueth et al. 1996; Ladd \& Hodapp 1997). Hence, time variability in velocity and/or direction is an important ingredient in the formation of molecular flows.

\subsubsection{Velocity Variability}

The effect of velocity variability of supersonic amplitude in YSO jets has been the object of numerous studies (see e.g. Raga 1993 for a review). As faster jet material catches up with slower, previously ejected gas, a two-shock structure develops (internal working surface), which propagates along the jet and drives a secondary bow shock into the low-density cocoon. Raga \& Cabrit (1993) showed analytically that its transverse size could be substantially larger than the jet radius and suggested that it could explain the low collimation of molecular flows. In particular, if the cocoon density drops with distance as $1 / d^{2}$, the secondary bow shock radius would grow linearly with $d$, reproducing the observed conical outflow shapes. At the same time, secondary bow shocks would produce local re-acceleration at positions of EHV features (which would trace the internal working surfaces in the jet). However, their simple analytical model did not include the confining effect of the main shell created by the leading jet head.

A 2D numerical simulation of a variable velocity jet in a molecular medium of decreasing density is shown in Fig. $4 . \mathrm{H}_{2}$ destruction and cooling are included (cf. Raga et al. 1995). While local forward reacceleration of the cocoon clearly occurs at each internal working surface, the sideways expansion of secondary bow shocks is indeed severely limited by the dense layer created by the leading jet head, and at this stage the flow is not much broader than without time variability (a similar result is found for a constant ambient density by Suttner et al. 1997). However, the decreasing environmental density gradient broadens the overall cavity at its end compared with the case of a uniform ambient medium (see also Gouveia dal Pino \& Birkinshaw 1996). The bow shock shape and its ratio to jet size are in striking agreement with e.g. the HH 211 flow (Guilloteau et al., this volume). Furthermore, the full opening angle at the origin increases with time, reaching $20^{\circ}$ in the last panel of Fig. 4 . Therefore, it is not excluded that the bow shock could eventually reach the full opening angles of $30-45^{\circ}$ typically observed in flows (Bachiller et al. 1995; Chandler et al. 1996 and references therein). In particular, the broadening effect of internal working surfaces may reveal itself on longer time scales than considered in current simulations, and needs further investigation. 

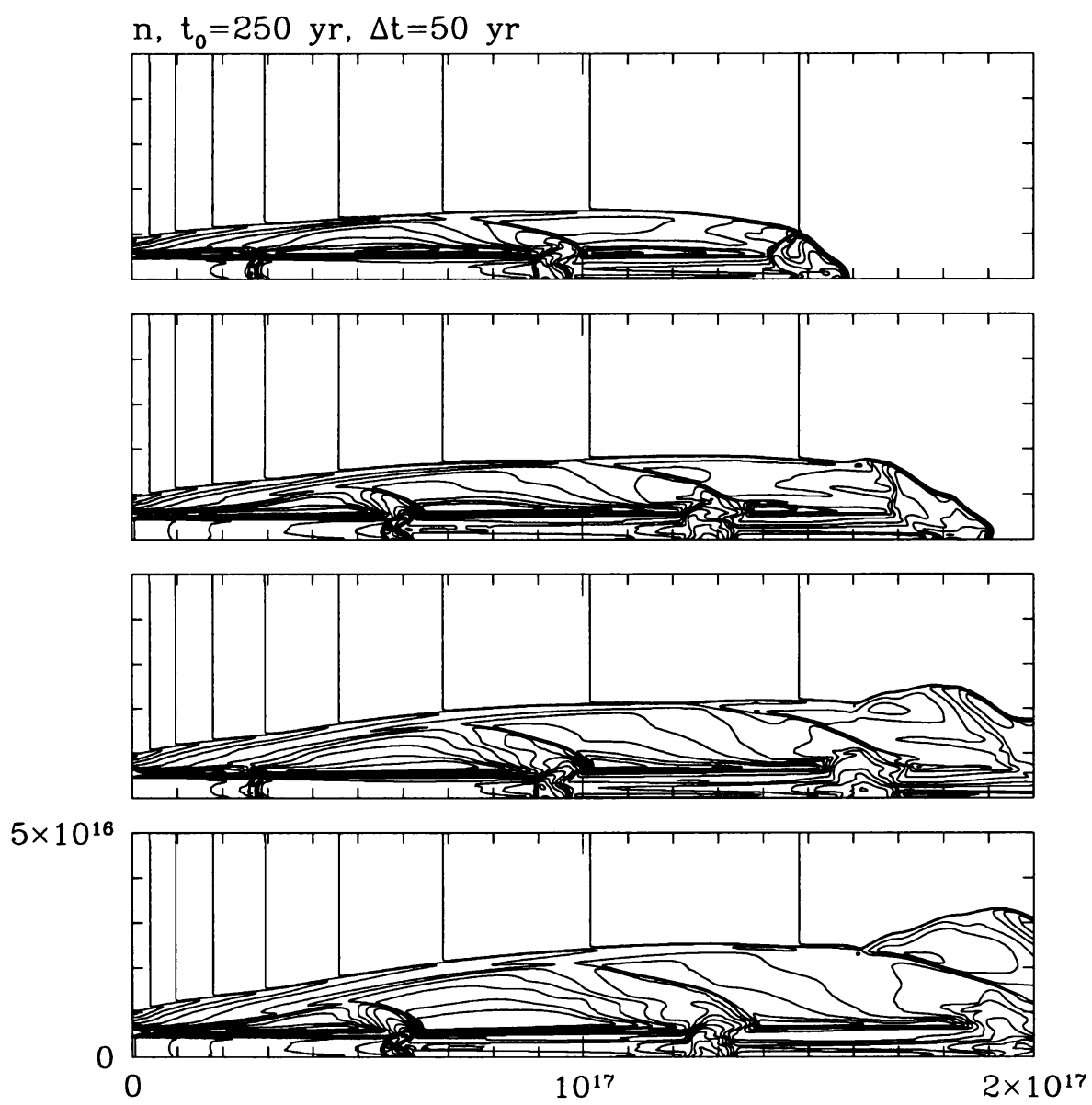

Figure 4. Total density at four time steps in a molecular jet with time-variable ejection velocity $\left(V_{j}(\mathrm{~km} / \mathrm{s})=150+50 \sin (2 \pi t / 100 \mathrm{yrs})\right)$, from Cabrit \& Raga (1997). Each internal working surface ejects material sideways, driving a new bow shock into the ambient cocoon. Molecular cloud density decreases outward as $\rho_{e}=\rho_{j} \times\left(1+x / 10^{16} \mathrm{~cm}\right)^{-2}$.

Another effect is noteworthy: at a given distance from the star, secondary bow shocks grow broader at each successive passage, as the main cavity continues expanding in the intervening time. If $\mathrm{CO}$ emission became dominated by one of the 'new' working surfaces, and not by the leading jet head, the observed collimation would be much lower than the true collimation of the full cavity. This is another illustration of the 'truncation' effect invoked above for explaining flow widths. 


\subsubsection{Directional Variability}

The propagation of a jet with continuously variable ejection direction was investigated analytically by Raga, Cantó \& Biro (1993). At growing distance from the source, the jet beam becomes increasingly misaligned with the direction of propagation. A sideways bow shock is driven into the environment and a jet shock into the inclined jet beam. In $2 \mathrm{D}$, a series of alternating one-sided bow shocks is formed (Biro et al. 1995); non-axisymmetric $\mathrm{K}-\mathrm{H}$ instabilities could produce similar structures (Stone et al. 1997). In precessing 3D jets, the spiral geometry is more complex (Cliffe et al. 1996). In either case, the jet 'effective radius' of interaction is increased, enhancing entrainment of surrounding gas, and the overall cavity could be substantially broadened for large precession angles (Cliffe et al. 1996).

Fully decoupled cavities at different angles, as observed in L1157 and Cep E (Gueth et al. 1996; Ladd \& Hodapp 1997), could be obtained with a combination of both velocity and directional variability. As shown analytically and numerically by Raga \& Biro (1993), the jet beam eventually breaks at the positions of the internal working surfaces, for some parameters giving rise to independent jet-bullets travelling in different directions, which interact with the environment through their own individual bow shock. Bullet deceleration is then expected, and may be seen in e.g. L1448 (Bachiller et al. 1990) and HH34 (Devine, 1997).

\section{Conclusions}

We have compared various models with the observed properties of outflows. Detailed morphologies and PV diagrams appear to provide stronger constraints than the global mass distribution with velocity. In particular, turbulent mixing layers along jet walls appear too narrow and/or decelerated to explain most outflows. The 'infall-driven' model of Fiege \& Henriksen $(1996 \mathrm{a}, \mathrm{b})$ is promising, but needs inclusion of non-radiative acceleration and demonstration of similar infall and outflow mass flux rates. Winddriven shells and jet-driven bow shocks remain so far the most appealing explanations for molecular outflows. Both models, though, currently have weak points that preclude a universal interpretation of observations. Possibly, each of these two models may apply to a different stage of YSO evolution or to a different luminosity range. Several important areas for future modelling work can be identified.

Wind-driven flows have so far been studied mostly through analytical work. Simple models are successful at reproducing the shape and integrated line profile of observed flows, but they meet several limitations. For example, radially expanding shells require nearly evacuated polar regions over large distances, and lack low-velocity emission at small view angles. How- 
ever, these predictions rely on extreme simplifying assumptions. Realistic numerical simulations would be useful to (1) clarify the actual constraints on wind and ambient density stratification; (2) see whether the shape, kinematics, and excitation of shock-heated molecular features at cavity ends can be reproduced; (3) investigate the effect of time variability for comparison with the multiple cavities now found in interferometric maps. In particular, cavities at different angles may be challenging to wind-driven shell models, which involve external collimation.

Jet-driven molecular bow shocks have been modelled numerically in increasing detail. They are very successful at explaining the velocity field of molecular outflows, and the associated shock-excited EHV features. Predicted $\mathrm{H}_{2}$ and $\mathrm{CO}$ emission maps are in striking agreement with the young flows discovered recently. However, bow shocks might produce too much transverse velocities, and lobes that remain too narrow (for typical jet radii). Both problems have possible solutions that need further theoretical backing: (1) the small amount of overlapping blue/red emission could be explained by very efficient turbulent mixing in the bow shock. It would be desirable to further study this effect; (2) broader, moderate collimation outflows might be obtained through a combination of time variability in velocity and direction (increasing the effective jet radius), and outflow 'truncation' (the outer parts leave the cloud and become unobservable, e.g. HH111; Cernicharo \& Reipurth 1996). These effects, and the long term evolution of bow shocks, need to be better understood and quantified with realistic parameters.

Finally, the wind-driven and bow shock models discussed here ignore magnetic fields, although they could have a substantial effect on the shock structure and emission properties (cf. Hollenbach; Pineau des Forêts \& Flower, this volume). This conference has seen several explorations of jet propagation in the presence of a magnetic field, and our understanding should progress substantially in the near future.

Future observations should also bring crucial informations on the formation processes of molecular outflows. More statistics on the true velocity range in flows, in particular at low inclinations, would provide interesting constraints. For such studies, shock-enhanced species (e.g. $\mathrm{SiO}$ ) will prove very useful. Unbiased large-scale mapping beyond known outflow limits, such as presented by Padman et al. (this volume), are also necessary to determine true flow extents and ages, and build an evolutionary sequence. Most importantly, interferometric high-resolution mapping is now revealing the inner structure (e.g. the multiple cavities and their actual morphologies) of a growing number of flows, and bring in some cases unique information on jet sizes and molecular content (Guilloteau et al., this volume). More observations with such high angular resolution are needed, to allow direct 
comparisons with numerical simulations.

Clearly, many issues regarding the formation and propagation of molecular outflows are still not settled, However, present ideas on this subject are quite promising. It will be necessary to focus on carrying out close comparisons between theoretical models and observations of selected flows, in order to narrow down the still quite wide range of possible mechanisms for explaining molecular outflows.

\section{References}

Bachiller, R. 1996, ARAA, 34, 111

Bachiller, R., Martín-Pintado, J., Tafalla, M., et al., 1990, A\&A, 231, 174

Bachiller, R., Guilloteau, S., Dutrey, A., Planesas, P., Martín-Pintado, J., 1995, A\&A, 299, 857

Bence, S.J., Richer, J.S., Padman, R., 1996, MNRAS, 279, 866

Barral, J.F., Cantó, J., 1981, RevMexAA, 5, 101

Biro, S., Raga, A.C., Cantó, J., 1995, MNRAS, 275, 557

Bontemps, S., André, P., Terebey, S., Cabrit, S. 1996, A\&A, 311, 858

Cabrit, S., Bertout, C., 1986, ApJ, 307, 313

Cabrit, S., Bertout, C., 1990, ApJ, 348, 530

Cabrit, S., Goldsmith, P.F., Snell, R.L., 1988, ApJ, 334, 196

Cabrit, S., Raga, A.C., 1997, in preparation

Cantó, J., 1980, A\&A, 86, 327

Cantó, J., Raga, A.C., 1991, ApJ, 372, 646

Cantó, J., Tenorio-Tagle, G., Rózyczka, M., 1988, A\&A, 192, 287

Cernicharo, J., Reipurth, B. 1996, ApJ, 460, L57

Chandler, C., Terebey, S., Barsony, M., Moore, T.J.T., Gautier, T.N, 1996, ApJ, 471, 308

Chernin, L.M., Masson, C.R., Gouveia dal Pino, E.M., Benz, W., 1994, ApJ, 426, 204

Cliffe, J.A., Franck, A., Jones, T.W., 1996, MNRAS, 282, 1114

Davis, C.J., Eislöffel, J., 1995, A\&A, 300, 851

Devine, D. 1997, in Low Mass Star Formation - from Infall to Outflow, eds. F. Malbet and A. Castets, p.95

Downes, T.P. 1996, in Solar and Astrophysical MHD flows, ed. K. Tsinganos (Kluwer)

Downes, T.P., Ray, T. Drury, L. 1997, in Low Mass Star Formation - from Infall to Outflow, eds. F. Malbet and A. Castets, p. 98

Dutrey, A., Guilloteau, S., Bachiller, R., 1997, A\&A, in press

Dyson, J.E., Cantó, J., Rodriguez, L.F., 1988, in Mass Outflows from Stars and Galactic Nuclei, L. Bianchi and R. Gilmozzi, ed(s)., Kluwer, Dordrecht, p. 299

Dyson, J. E., Hartquist, T. W., Malone, M. T., and Taylor, S. D. 1995, RevMexAA Conf. Series, 1, p. 119.

Eislöffel, J., Davis, C.J., Ray, T.P., Mundt, R., 1994, ApJ, 422, L91

Fiege, J.D., Henriksen, R.N., 1996a, MNRAS, 281, 1038

Fiege, J.D., Henriksen, R.N., 1996b, MNRAS, 281, 1055

Frank, A., Mellema, G., 1996, ApJ, 472, 684

Fuller, G.A., Ladd, E.F., Padman, R., Myers, P.C., Adams, F. C. 1995, ApJ, 454, 862

Gouveia dal Pino, E.M., Birkinshaw, M., 1996, ApJ, 471, 832

Gredel, R., Reipurth, B., 1993, ApJ, 407, L29

Gueth, F., Guilloteau, S., Bachiller R., 1996, A\&A, 307, 891

Guilloteau, S., Bachiller, R., Fuente, A., Lucas, R., 1992, A\&A, 265, L49

Hartigan, P., Patrick, R.J., Hartmann, L., 1987, ApJ, 316, 323

Hartigan, P., Morse, J., Raymond, J., 1994, ApJ, 436, 125 
Henriksen, R.N., Valls-Gabaud D., 1994, MNRAS, 266, 681

Holland, W.S., Greaves, J.S., Ward-Thompson, D., André, P., 1996, A\&A, 309, 267

Königl, A., 1982, ApJ, 261, 115

Koo, B.C., McKee C.F., 1992, ApJ, 388, 93

Ladd, E.F., Hodapp K.W., 1997, ApJ, 474, 749

Lada, C.J., 1985, ARAA, 23, 267

Lada, C.J., Fich M., 1996, ApJ, 459, 638

Levreault, R.M., 1988, ApJ, 330, 897

Li, Z.Y., Shu F.H., 1996, ApJ, 472, 211

Lizano, S., Heiles, C., Rodriguez L.F., et al., 1988, ApJ, 328, 763

Lizano, S., Giovanardi, C., 1995, ApJ, 447, 742.

Masson, C.R., Chernin, L.M., 1992, ApJ, 387, L47

Masson, C.R., Chernin, L.M., 1993, ApJ, 414, 230

Masson, C.R., Mundy, L.G., Keene, J., 1990, ApJ, 357, L25

McCaughrean, M.J., Rayner, J.T., Zinnecker, H., 1994, ApJ, 436, L189

Mellema, G., Frank, A., 1995, ApSS 233, 145

Meyers-Rice, B.A., Lada, C.J., 1991, ApJ, 368, 445

Moriarty-Schieven, G.H., Snell, R.L., 1988, ApJ, 332, 364

Mundt, R., Brugel, E.W., Bührke, E., 1987, ApJ, 319, 275

Nagar N.M., Vogel, S.N., Stone, J.M., Ostriker, E.C., 1997, ApJL, in press

Padman, R., Richer, J.S., 1994, ApSS, 216, 129

Raga, A.C., 1993, ApSS, 208, 163

Raga, A.C., 1995, RevMexAA Conf. Series, 1, p. 103

Raga, A.C., Cantó, J. 1997, in Open problems on astrophysical jets, eds. S. Massaglia and G. Bodo (Gordon and Breach, in press).

Raga, A.C., Biro, S., 1993, MNRAS, 264, 758

Raga, A.C., Cabrit, S., 1993, A\&A, 278, 267

Raga, A.C., Cantó, J., Biro, S., 1993, MNRAS, 260, 163

Raga, A.C., Cantó, J., Calvet., N., Rodriguez, L.F., Torrelles., J.M., 1993, A\&A, 276, 539

Raga, A.C., Cabrit, S., Cantó, J., 1995, MNRAS, 273, 422

Raga, A.C., Taylor, S.D., Cabrit, S., Biro, S., 1995, A\&A, 296, 833

Richer, J.E., Hills, R.E., Padman, R., 1992, MNRAS, 254, 525

Rodríguez, L.F., Carral, P., Moran, J.M., Ho, P.T.P. 1982, ApJ, 260, 635

Shu, F.H., Ruden, S.P., Lada, C.J., Lizano, S., 1991, ApJ, 370, L31

Shu, F.H., Najita, J., Ostriker, E.C., Shang, H, 1995, ApJ, 455, L155

Smith, M.D, 1986, MNRAS, 223, 57

Smith, M.D., Brand, P., Moorhouse, A., 1991, MNRAS, 248, 451

Smith, M.D., Suttner, G., Yorke, H.W., 1997, A\&A, in press

Smith, M.D., Smarr, L., Norman, M.L., Wilson, J.R. 1983, ApJ, 264, 432

Snell, R.L., Loren, R.B., Plambeck, R.L, 1980, ApJ, 239, 17

Stahler, S., 1994, ApJ, 422, 616

Stone, J.M., Xu, J., Hardee, P.E. 1997, ApJ, in press

Suttner G., Smith, M.D., Yorke, H.W., Zinnecker, H., 1997, A\&A, in press

Taylor, S., Raga, A.C., 1995, A\&A, 296, 823

Wilkin, F.P., 1996, ApJ, 459, 31

Wilkin, F.P., et al. 1997, in preparation

Wolfire, M.G., Königl, A., 1991, ApJ, 383, 205

Xie, T., Goldsmith, P.F., Patel, N, 1993, ApJ, 419, 33 